\title{
Presencia de anticuerpos frente a algunos patógenos de interés zoonótico en cuatro granjas porcícolas de Cundinamarca, Colombia
}

\author{
Presence of antibodies against some pathogens of zoonotic interest in \\ four pig farms of Cundinamarca, Colombia
}

\author{
Adriana Pulido-Villamarín ${ }^{1,2}$, Rubiela Castañeda-Salazar ${ }^{1}$, \\ María Fernanda Mendoza-Gómez ${ }^{1}$, Laura Vivas-Díaz ${ }^{1}$
}

\section{Resumen}

El objetivo del presente trabajo fue determinar la presencia de anticuerpos frente a patógenos de cerdos que representan riesgo en salud pública por su potencial zoonótico (Salmonella spp, Leptospira interrogans, Yersinia spp, Trichinella spp y Toxoplasma gondii) en sueros porcinos obtenidos en cuatro granjas porcícolas semi-tecnificadas de Cundinamarca, Colombia. Se colectaron 89 muestras de sangre y se analizaron utilizando los estuches de ELISA para diagnóstico Pigtype ${ }^{\circledR}$-Salmonella Ab, Pigtype ${ }^{\circledR}-$ Yopscreen, Pigtype $^{\circledR}-$ Toxoplasma Ab y Pigtype ${ }^{\circledR}$-Trichinella $\left(\right.$ QuiaGen $\left.^{\circledR}\right)$. La detección de anticuerpos frente a Leptospira spp se realizó mediante la prueba de microaglutinación lisis (MAT). La presencia de anticuerpos frente a Salmonella spp se detectó en el $40 \%$ de las muestras, mientras que para Yersinia spp y Toxoplasma gondii fue en el 18 y $1.1 \%$ respectivamente. Todas las granjas fueron positivas a por lo menos uno de los 13 serovares de Leptospira interrogans y ninguna de las muestras fue positiva para Trichinella spp. Se concluye que los cerdos del estudio han tenido contacto en algún momento de su ciclo productivo con la mayoría de los patógenos zoonóticos evaluados, lo que puede representar un riesgo para la salud pública y la producción porcícola.

Palabras clave: zoonosis; Salmonella spp; Leptospira interrogans; Yersinia spp; Trichinella spp; Toxoplasma gondii; porcinos; Colombia

${ }^{1}$ Línea de Epidemiología y Salud Animal, UNIDIA, Departamento de Microbiología, Facultad de Ciencias, Pontificia Universidad Javeriana, Bogotá, Colombia

${ }^{2}$ E-mail: adriana.pulido@javeriana.edu.co

Recibido: 26 de julio de 2018

Aceptado para publicación: 2 de enero de 2019 
The aim of this study was to determine the presence of antibodies against pathogens of pigs that represent public health risk due to their zoonotic potential (Salmonella spp, Leptospira interrogans, Yersinia spp, Trichinella spp and Toxoplasma gondii) in porcine sera obtained in four semi-technified pig farms from Cundinamarca, Colombia. A total of 89 blood samples were collected and analyzed using the ELISA diagnostic kits Pigtype ${ }^{\circledR}-$ Salmonella Ab, Pigtype ${ }^{\circledR}$-Yopscreen, Pigtype ${ }^{\circledR}-$ Toxoplasma Ab and Pigtype ${ }^{\circledR}-$ Trichinella (QuiaGen $\left.{ }^{\circledR}\right)$ The detection of antibodies against Leptospira spp was carried out by the microagglutination lysis test (MAT). Antibodies against Salmonella spp was observed in $40 \%$ of the samples, while for Yersinia spp and Toxoplasma gondii was in 18 and $1.1 \%$ respectively. At least one of the 13 serovars of Leptospira interrogans was detected in all four farms and none of the samples was positive for Trichinella spp. It is concluded that the pigs in the study had contact at some point of their productive cycle with most of the zoonotic pathogens evaluated, which may represent a risk to public health and pig production.

Key words: zoonosis, Salmonella spp; Leptospira interrogans; Yersinia spp; Trichinella spp; Toxoplasma gondii; pigs; Colombia

\section{INTRODUCCIÓN}

La industria porcícola colombiana ha evidenciado en los últimos 10 años un notable y constante crecimiento. En el primer trimestre de 2017 el sacrificio de ganado porcino fue de 946425 cabezas, comparado con las 894483 cabezas para el mismo periodo del año anterior (DANE, 2017); por otra parte, el consumo per cápita ha incrementado hasta casi duplicarse en la última década, llegando a los $6.6 \mathrm{~kg} /$ habitante/año (DANE, 2017), dejando de lado la estigmatización de hace algunos años con respecto a este tipo de alimento, tanto a nivel nacional como internacional (FAO, 2014b). Sin embargo, las ganaderías porcícolas son susceptibles de ser afectadas por patógenos como virus, parásitos y bacterias, que traen consigo pérdidas económicas y graves problemas sanitarios, además que algunos de estos pueden representar riesgos para la salud pública por ser reconocidos como patógenos zoonóticos transmitidos por alimentos (Baer et al., 2013; FAO, 2014a). Algunos de estos son Salmonella spp, Leptospira spp, Yersinia spp, Trichinella spp y Toxoplasma gondii (Solaymani-Mohammadi y Petri, 2006; Baer et al., 2013).

De acuerdo con la FAO, enfermedades como la triquinosis y la yersiniosis están asociadas a sistemas de producción extensivos y de baja inversión, en los cuales las medidas sanitarias y de higiene son deficientes; por otro lado, las intoxicaciones alimentarias producidas por Salmonella spp no se encuentran asociadas de manera específica a determinados sistemas de producción, por lo que son consideradas como un motivo de preocupación mundial (FAO, 2014a). Adicionalmente, según la clasificación mundial de parásitos transmitidos por alimentos, Toxoplasma gondii y Trichinella spiralis ocupan el $4^{\circ}$ y $7^{\circ}$ puesto respectivamente, donde la carne de cerdo juega un papel importante en la transmisión de agentes zoonóticos (FAO/WHO, 2014). Por otra parte, la leptospirosis es una enfermedad de distribución mundial de gran importancia por las altas tasas de mortalidad que ocasiona en humanos y animales, con reportes anuales de 500000 casos severos y mortalidad superior 
al 10\% en humanos (García et al., 2013); así, en 2016 en Colombia fueron confirmados 562 casos de leptospirosis (INS-Sivigila, 2016); además de producir grandes pérdidas económicas en las producciones porcícolas (Ochoa et al., 2000).

Según los reportes del Instituto Colombiano Agropecuario (ICA) en 2014 para el sector porcícola nacional, la mortalidad ocasionada por salmonelosis fue de $1.5 \times 1000$, viéndose afectadas 21 granjas, con una población en riesgo de 14248 animales y datos de incidencia del 1\%. Casos de leptospirosis fueron reportados en 25 explotaciones con una población en riesgo de 34751 porcinos y datos de incidencia del 1\%, sin casos de mortalidad (Díaz et al., 2014). Yersinia spp, $T$. gondii y Trichinella spp no han sido reportados en el sector porcícola colombiano por los entes regidores de la salud animal.

Teniendo en cuenta el riesgo al que se encuentra expuesta la población humana al entrar en contacto con porcinos infectados desde su labor ocupacional o al consumir carne de cerdo contaminada, es indispensable que desde la producción primaria se realicen pruebas diagnósticas para determinar, si el animal ha tenido contacto con patógenos en alguna fase de su vida, mediante la detección de anticuerpos circulantes, y de esta manera, implementar medidas de prevención y control en las granjas (FAO/WHO, 2014). Por lo anterior, el objetivo del presente estudio fue determinar la presencia de anticuerpos frente a Salmonella spp, Leptospira interrogans, Yersinia spp, Trichinella spp y Toxoplasma gondii en cerdos de ceba y hembras de cría de cuatro granjas porcícolas semi-tecnificadas de Cundinamarca, Colombia.

\section{Materiales y Métodos}

Se realizó un muestreo por conveniencia y se obtuvieron 89 muestras sanguíneas de porcinos en ceba (133-154 días, $\geq 105 \mathrm{~kg}$ ) y hembras de cría ( $\geq 1$ parto), distribuidos en cuatro granjas semi-tecnificadas $(\leq 200 \mathrm{hem}$ bras de cría) del departamento de Cundinamarca, Colombia (Cuadro 1). Las muestras se obtuvieron a partir de la vena yugular en tubos Vacutainer sin anticoagulante (Framstad et al., 2000; Casas, 2013) y refrigeradas para su transporte al laboratorio en un tiempo no mayor a tres horas.

Las muestras fueron centrifugadas a $1500 \mathrm{~g}$ por 5 minutos para la obtención del suero. Estos fueron procesados mediante la técnica de ELISA indirecta utilizando los estuches Salmonella Pigtype ${ }^{\circledR}$-Salmonella Ab, Pigtype $^{\circledR}$-Yopscreen para Yersinia spp, Pigtype $^{\circledR}-$ Toxoplasma Ab para Toxoplasma gondii y Pigtype $^{\circledR}$-Trichinella para Trichinella spp (QuiaGen ${ }^{\circledR}$ ), siguiendo las indicaciones de la casa comercial. Específicamente, el estuche Salmonella Pigtype $^{\circledR}$-Salmonella Ab permite detectar anticuerpos contra los serotipos pertenecientes a los grupos B, C, D, y E, donde se encuentran los serovares patógenos para humanos y el Pigtype ${ }^{\mathbb{}}-$ Yopscreen permite detectar anticuerpos contra la membrana externa de Yersinia spp de cepas patógenas. La sensibilidad y especificidad reportada para estos estuches comerciales es del $97 \%$. La técnica de microaglutinación lisis (MAT) para 13 serovares de Leptospira interrogans (Cuadro 2), fue realizada siguiendo el protocolo establecido por la OMS (2008).

La interpretación de resultados para las pruebas de ELISA se realizó de acuerdo con las indicaciones de la casa productora, teniendo en cuenta la densidad óptica (DO) reportada para cada una de las muestras con respecto a las DO de los controles. El cociente $\mathrm{S} / \mathrm{P}$ fue calculado como $\left(\mathrm{OD}_{\text {sample }}-\mathrm{MV}\right.$ $\mathrm{OD}_{\mathrm{NC}}$ ) / $\mathrm{MV} \mathrm{OD} \mathrm{PC}_{\mathrm{PC}}-\mathrm{MV} \mathrm{OD}_{\mathrm{NC}}$, donde MV: valor de la media, NC: control negativo, $\mathrm{PC}$ : control positivo. Se consideraron como muestras positivas aquellas con un cociente $\mathrm{S} / \mathrm{P}$ mayor o igual a 0.3 y como muestras negativas aquellas con un cociente S/P menor a 0.3 . 
Cuadro 1. Distribución de las 89 muestras de sangre obtenidas en las cuatro granjas porcinas según el sexo del cerdo (Cundinamarca, Colombia)

\begin{tabular}{lcccc}
\hline & $\begin{array}{c}\text { Granja 1 } \\
\text { (Municipio de } \\
\text { Santandercito) }\end{array}$ & $\begin{array}{c}\text { Granja 2 } \\
\text { (Municipio de } \\
\text { Ubaté) }\end{array}$ & $\begin{array}{c}\text { Granja 3 } \\
\text { (Municipio de } \\
\text { Chocontá) }\end{array}$ & $\begin{array}{c}\text { Granja 4 } \\
\text { (Municipio de } \\
\text { Facatativá) }^{(a)}\end{array}$ \\
\hline Machos & 5 & 6 & 11 & 10 \\
Hembras & 17 & 17 & 10 & 13 \\
\hline Total & 22 & 23 & 21 & 23 \\
\hline
\end{tabular}

Granja 1: $4^{\circ} 36^{\prime} 58^{\prime \prime} \mathrm{N} 74^{\circ} 21^{\prime} 08^{\prime \prime} \mathrm{O}$; Granja 2: $5^{\circ} 18^{\prime} 26^{\prime \prime} \mathrm{N} 73^{\circ} 48^{\prime} 52^{\prime \prime} \mathrm{O}$; Granja 3: $5^{\circ} 08^{\prime} 48^{\prime \prime} \mathrm{N} 73^{\circ} 40^{\prime} 57^{\prime \prime} \mathrm{O}$; Granja 4: $4^{\circ} 48^{\prime} 53^{\prime \prime} \mathrm{N} 74^{\circ} 21^{\prime} 19^{\prime \prime} \mathrm{O}$

(a) Se vacuna contra Leptospira interrogans

Cuadro 2. Títulos frente a Leptospira interrogans en tres granjas porcinas sin vacunación de tres municipios de Cundinamarca, Colombia

\begin{tabular}{|c|c|c|c|c|c|c|c|c|c|c|c|c|c|c|}
\hline \multicolumn{15}{|l|}{ Granja } \\
\hline & 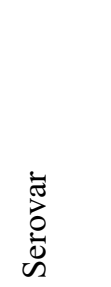 & $\begin{array}{l}\frac{\mathscr{n}}{\tilde{\sigma}} \\
\text { 言 } \\
\text { 至 }\end{array}$ & 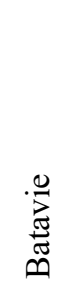 & 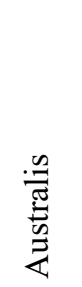 & 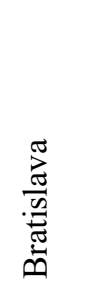 & $\begin{array}{l}\frac{\pi}{0} \\
.00 \\
\tilde{\Xi} \\
\tilde{\Xi}\end{array}$ & 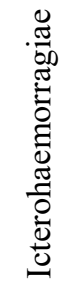 & 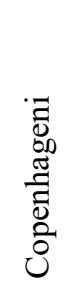 & 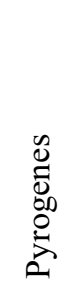 & 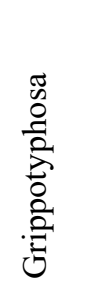 & 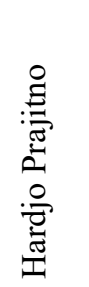 & $\dot{\Xi}$ & 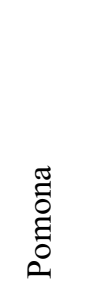 & 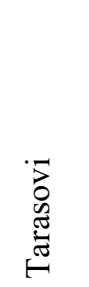 \\
\hline & Tít. ${ }^{1}$ & $\begin{array}{l}\% \\
(\mathrm{n}) \\
\end{array}$ & $\begin{array}{l}\% \\
(\mathrm{n})\end{array}$ & $\begin{array}{l}\% \\
(\mathrm{n})\end{array}$ & $\begin{array}{l}\% \\
(\mathrm{n})\end{array}$ & $\begin{array}{l}\% \\
(\mathrm{n}) \\
\end{array}$ & $\begin{array}{l}\% \\
(\mathrm{n})\end{array}$ & $\begin{array}{l}\% \\
(\mathrm{n})\end{array}$ & $\begin{array}{l}\% \\
(\mathrm{n})\end{array}$ & $\begin{array}{l}\% \\
(\mathrm{n})\end{array}$ & $\begin{array}{l}\% \\
(\mathrm{n})\end{array}$ & $\begin{array}{l}\% \\
(\mathrm{n}) \\
\end{array}$ & $\begin{array}{l}\% \\
(\mathrm{n}) \\
\end{array}$ & $\begin{array}{l}\% \\
(\mathrm{n}) \\
\end{array}$ \\
\hline \multirow{2}{*}{$\begin{array}{l}\text { Granja } \\
1 \\
(\mathrm{n}=22)\end{array}$} & $1: 50$ & $\begin{array}{l}9.0 \\
(2)\end{array}$ & 0 & $\begin{array}{l}4.5 \\
(1)\end{array}$ & $\begin{array}{l}4.5 \\
\text { (1) }\end{array}$ & $\begin{array}{l}4.5 \\
\text { (1) }\end{array}$ & 0 & $\begin{array}{l}4.5 \\
\text { (1) }\end{array}$ & 0 & 0 & $\begin{array}{l}9.0 \\
(2)\end{array}$ & $\begin{array}{l}4.5 \\
\text { (1) }\end{array}$ & 0 & 0 \\
\hline & $\geq 1: 100$ & 0 & 0 & 0 & 0 & $\begin{array}{l}4.5 \\
(1)\end{array}$ & $\begin{array}{l}4.5 \\
(1)\end{array}$ & 0 & 0 & $\begin{array}{l}4.5 \\
(1)^{(b)}\end{array}$ & 0 & 0 & 0 & 0 \\
\hline \multirow{2}{*}{$\begin{array}{l}\text { Granja } \\
2 \\
(\mathrm{n}=23)\end{array}$} & $1: 50$ & 0 & 0 & $\begin{array}{l}8.6 \\
(2)\end{array}$ & $\begin{array}{l}4.3 \\
\text { (1) }\end{array}$ & $\begin{array}{l}13.0 \\
\text { (3) }\end{array}$ & 0 & 0 & $\begin{array}{l}4.3 \\
(1)\end{array}$ & 0 & $\begin{array}{l}4.3 \\
\text { (1) }\end{array}$ & 0 & $\begin{array}{l}21.7 \\
(5)\end{array}$ & $\begin{array}{l}13.0 \\
\text { (3) }\end{array}$ \\
\hline & $\geq 1: 100$ & $\begin{array}{l}8.6 \\
\text { (2) }\end{array}$ & $\begin{array}{l}8.6 \\
(2)\end{array}$ & 0 & $\begin{array}{l}13.0 \\
(3)^{(b)}\end{array}$ & 0 & $\begin{array}{l}8.6 \\
(2)\end{array}$ & 0 & $\begin{array}{l}4.3 \\
\text { (1) }\end{array}$ & $\begin{array}{l}4.3 \\
\text { (1) }\end{array}$ & $\begin{array}{l}8.6 \\
(2)^{(b)}\end{array}$ & $\begin{array}{l}4.3 \\
\text { (1) }\end{array}$ & 0 & $\begin{array}{l}8.6 \\
(2)^{(b)}\end{array}$ \\
\hline \multirow{2}{*}{$\begin{array}{l}\text { Granja } \\
3(\mathrm{n}= \\
21)\end{array}$} & $1: 50$ & 0 & 0 & $\begin{array}{l}14.3 \\
\text { (3) }\end{array}$ & $\begin{array}{l}9.5 \\
(2)\end{array}$ & $\begin{array}{l}9.5 \\
(2)\end{array}$ & 0 & $\begin{array}{l}4.8 \\
\text { (1) }\end{array}$ & $\begin{array}{l}9.5 \\
(2)\end{array}$ & $\begin{array}{l}4.8 \\
\text { (1) }\end{array}$ & 0 & 0 & $\begin{array}{l}9.5 \\
(2)\end{array}$ & 0 \\
\hline & $\geq 1: 100$ & $\begin{array}{l}4.8 \\
(1) \\
\end{array}$ & 0 & $\begin{array}{l}9.5 \\
(2) \\
\end{array}$ & $\begin{array}{l}19.0 \\
(4) \\
\end{array}$ & $\begin{array}{l}4.8 \\
(1) \\
\end{array}$ & $\begin{array}{l}4.8 \\
(1) \\
\end{array}$ & 0 & 0 & $\begin{array}{l}9.5 \\
(2)^{(b)}\end{array}$ & $\begin{array}{l}14.3 \\
(3)^{(\mathrm{c})}\end{array}$ & $\begin{array}{l}4.8 \\
\text { (1) }\end{array}$ & $\begin{array}{l}4.8 \\
(1)\end{array}$ & $\begin{array}{l}4.8 \\
(1)\end{array}$ \\
\hline \multirow{2}{*}{$\begin{array}{l}\text { Total } \\
(\mathrm{n}=66)\end{array}$} & $1: 50$ & $\begin{array}{l}2.2 \\
\text { (2) }\end{array}$ & 0 & $\begin{array}{l}6.7 \\
(6)\end{array}$ & $\begin{array}{l}4.5 \\
(4)\end{array}$ & $\begin{array}{l}6.7 \\
(6)\end{array}$ & 0 & $\begin{array}{l}2.2 \\
(2)\end{array}$ & $\begin{array}{l}3.4 \\
\text { (3) }\end{array}$ & $\begin{array}{l}1.1 \\
\text { (1) }\end{array}$ & $\begin{array}{l}3.4 \\
(3)\end{array}$ & $\begin{array}{l}1.1 \\
(1)\end{array}$ & $\begin{array}{l}7.8 \\
(7)\end{array}$ & $\begin{array}{l}3.4 \\
\text { (3) }\end{array}$ \\
\hline & $\geq 1: 100$ & $\begin{array}{l}3.4 \\
\text { (3) }\end{array}$ & $\begin{array}{l}2.2 \\
(2)\end{array}$ & $\begin{array}{l}2.2 \\
(2)\end{array}$ & $\begin{array}{l}7.8 \\
(7)\end{array}$ & $\begin{array}{l}2.2 \\
(2)\end{array}$ & $\begin{array}{l}4.5 \\
(4)\end{array}$ & 0 & $\begin{array}{l}1.1 \\
(1)\end{array}$ & $\begin{array}{l}4.5 \\
(4)\end{array}$ & $\begin{array}{l}5.6 \\
(5)\end{array}$ & $\begin{array}{l}2.2 \\
(2)\end{array}$ & $\begin{array}{l}1.1 \\
(1)\end{array}$ & $\begin{array}{l}3.4 \\
(3)\end{array}$ \\
\hline
\end{tabular}

Granja 1 (Municipio de Santandercito), Granja 2 (Municipio de Ubaté), Granja 3 (Municipio de Chocontá)

${ }^{1}$ Título; ${ }^{b}$ Una muestra con título 1:200; ${ }^{c}$ Dos muestras con título 1:200 
En la prueba MAT, los títulos $\geq 1: 100$ son considerados como positivos, indicando un posible contacto previo, la posible presencia de infección actual o anticuerpos vacunales en disminución (Picardeau, 2013; Calderón et al., 2014). Los títulos de 1:50 podrían sugerir que los animales tuvieron algún tipo de contacto previo con la bacteria y los títulos $\leq 1: 25$ indican títulos de anticuerpos no detectables.

\section{Resultados}

\section{Salmonella spp}

El 40\% (36/89) de las muestras fueron positivas a Salmonella spp. Los resultados individuales por granja evidenciaron que la granja 3 fue la de mayor seropositividad con $57.1 \%(\mathrm{n}=12)$, seguida de las granjas 1 y 4 con $40.9 \%(n=9)$ y $39.1 \%(n=9)$, respectivamente. La granja 2 fue la de menor seropositividad con el $26.1 \%(n=6)$.

\section{Yersinia spp}

El 18\% (16/89) de los sueros evaluados fueron positivos a Yersinia spp. Las granjas 1,3 y 4 fueron negativas para el patógeno, mientras que en la Granja 2 se obtuvo una positividad del $69.6 \%$.

\section{Leptospira interrogans}

Todas las granjas presentaron positividad por los menos a uno de los serovares evaluados. Los resultados discriminados para las granjas sin vacunación (1, 2 y 3$)(n=66)$ se presentan en el Cuadro 2, donde se evidencia mayor seropositividad para el serovar $L$. Bratislava (título $\geq 1: 100$ ) con $7.8 \%$ (Cuadro 2).

En la granja $4(n=23)$ de animales vacunados se detectaron títulos de anticuerpos de 1:100 para los serovares $L$. Autumnalis (4.3\%) y $L$. Bratislava (8.7\%).

\section{Toxoplasma spp y Trichinella spp}

Solo se encontró una muestra positiva (1.1\%) positiva para Toxoplasma spp correspondiente a una hembra de la granja 1.

\section{Discusión}

El conocimiento de la situación sanitaria frente a patógenos zoonóticos presentes en las granjas porcícolas favorece la implementación de medidas de prevención y control tendientes al mejoramiento del estado sanitario de los animales, así como de los parámetros productivos de la granja. Esto puede contribuir a la disminución de su transmisión al humano; siendo la serología una herramienta de monitoreo que permite conocer la situación de los animales frente a la presencia y posible exposición a estos patógenos en las granjas.

La carne de cerdo es una fuente reconocida de Salmonella spp, por lo que la determinación de anticuerpos en suero permite predecir el riesgo de contaminación de la canal (van Der Wolf et al., 2001; Bonde y Sorensen, 2012). En el presente estudio, los valores de anticuerpos se encuentran dentro de los niveles reportados para los Países Bajos, Rumania y España (23.7-73.4\%) y en general para la Unión Europea (81-93\%) (van Der Wolf et al., 2001; Hernández et al., 2014; Surpat et al., 2014; Gradassi et al., 2015). Los corrales con piso de cemento favorecen un mayor contacto con las heces, representando un factor de riesgo importante para la transmisión oro-fecal entre animales (van Der Wolf et al., 2001), lo que sumado a la posible presencia de aves y roedores, y al tipo de agua utilizada en la granja se constituyen en los principales factores de riesgo reportados en la porcicultura colombiana para la infección con Salmonella spp en los animales (Henao et al., 2012). 
El cerdo es reconocido como el principal reservorio de $Y$. enterocolitica bioserotipo 4/O:3 (Von Altrock et al., 2011; LaukknenNinios et al., 2014), de modo que la contaminación de la carne destinada al consumo humano representa un riesgo para la salud pública. Estudios realizados en granjas y plantas de beneficio en Bélgica, Italia y Alemania determinaron una seroprevalencia del $66 \%$ (Vanantwerpen et al., 2014), 56.1\% (Bonardi et al., 2016) y $64.1 \%$ (Von Altrock et al., 2011), respectivamente; valores altos con respecto al 18\% detectado en el presente estudio. Los factores de riesgo asociados a la infección con este microorganismo son los mismos mencionados para Salmonella spp. Cabe resaltar que las granjas analizadas reportaron el cumplimiento adecuado de las buenas prácticas de manejo (BPM) al contar con un sistema todo dentro/todo fuera, realizar procesos de limpieza y desinfección de corrales una vez al día, aunque el agua utilizada no proviene de una planta de tratamiento y, por lo tanto, se desconoce su calidad; por otro lado, aunque se reporta la presencia de roedores, existe un programa de manejo integrado de plagas (MIP).

En la evaluación de los títulos de anticuerpos frente a 13 serovares de $L$. interrogans, se detectó que las cuatro granjas presentaron positividad a por lo menos uno de los serovares evaluados, con predominio de $L$. Bratislava y $L$. Pomona (Cuadro 2). Estos hallazgos coinciden con reportes de literatura, donde los serovares generalmente asociados a porcinos son Pomona, Bratislava, Canicola, Icterohaemorrhagiae y Grippotyphosa (Vado-Solis et al., 2002; Feraud y Abeledo, 2005; Alder y de la Peña, 2010; Dechner, 2014), aunque también han sido reportados Tarassovi, Copenhague, Hardjo, Panama, Shermani, Pyrogenes, Australis y Wolffi, cuya presencia se ha relacionado con factores como prácticas de manejo y bioseguridad, tipo de explotación, esquema de inmunización, introducción de animales nuevos y, especialmente, por contacto con otras especies animales como caninos, bovinos y roedores (Vado-Solis et al., 2002;
Feraud y Abeledo, 2005). Basados en esto y considerando que el principal serovar asociado en Colombia a los porcinos es Pomona y en roedores es Icterohaemorragiae (Almenteros et al., 2004; Romero-Vivas et al., 2013), se podría sugerir que la presencia de estos últimos en las granjas ha podido favorecer la presencia de los serovares Icterohaemorragiae y Grippotyphosa en las granjas 1, 2 y 3 , y Bratislava en las granjas 2 y 3 . Adicionalmente, el serovar Grippotyphosa ha sido reportado en caninos, de modo que su presencia en las granjas podría contribuir al riesgo de infección para los porcinos (Calderón et al., 2014). Los resultados indican la circulación de múltiples serovares en las granjas porcícolas.

En el caso de la granja 4 con esquema de vacunación para Leptospira spp se encontraron solo dos muestras con títulos $>1: 100$ para el serovar Bratislava. Es importante considerar que la mayoría de las vacunas disponibles para porcinos en el mercado contienen al menos los serovares Pomona, Canicola, Grippotyphosa, Bratislava e Icterohaemorrhagiae (Alder y de la Peña, 2010). Por otro lado, una sola muestra para la determinación de anticuerpos mediante MAT no permite diferenciar entre anticuerpos vacunales y anticuerpos producidos por la infección con el agente infeccioso.

En el porcino, la infección con T. gondii y Trichinella spp cursa de manera subclínica; sin embargo, a nivel de salud pública los abortos, malformaciones en neonatos y secuelas neurológicas en personas adultas cobran importancia en la infección por $T$. gondii, mientras que la infección con Trichinella spp puede causar enfermedad aguda, muchas veces fatal (FAO/WHO, 2014). Los resultados obtenidos evidencian una baja exposición de los animales a $T$. gondii (1.1\%), hallazgos similares a los reportados en los Países Bajos (2.6\%). Sin embargo, Herrero et al. (2016) reportaron $24.5 \%$ de seroprevalencia mediante la técnica de inmunofluorescencia indirecta en muestras de sueros de 1200 cerdos de 161 granjas en Aragón, España. 
Adicionalmente, se pueden encontrar seroprevalencias mayores al $95 \%$ en países como Estados Unidos y México (Lehmann et al., 2003; Hill et al., 2006; Ortega-Pacheco et al., 2013).

No se detectó exposición a Trichinella spp en las granjas analizadas, lo que coincide con las seroprevalencias halladas en España (0\%) (Hernández et al., 2014) y en Países Bajos (0.24\%) (van Der Giessen et al., 2007). Trichinella spp no ha sido detectado a la fecha en Colombia (Laverde et al., 2009), aunque existen reportes de su presencia en varios países de la región (Pozio, 2007).

\section{Conclusiones}

- Los cerdos de las cuatro granjas de Cundinamarca, Colombia, pueden haber estado en contacto con patógenos zoonóticos como Salmonella spp, Leptospira interrogans, Yersinia spp y Toxoplasma gondii, lo que puede representar un riesgo para la salud pública y la producción porcícola.

- No se detectó la presencia de anticuerpos contra Trichinella spp.

\section{Agradecimientos}

A Quimiolab S.A.A, por la donación de los estuches de ELISA Quiagen ${ }^{\circledR}$ para diagnóstico y a la Asociación Colombiana de Porcicultores (PorkColombia) por el apoyo económico para la realización de las pruebas de microglutinación lisis para Leptospira spp. Así mismo, a la Pontificia Universidad Javeriana.

\section{Literatura Citada}

1. Adler B, de la Peña-Moctezuma A. 2010. Leptospira and leptospirosis. Vet Microbiol 140: 287-296. doi: 10.1016/ j.vetmic.2009.03.012
2. Almenteros C, Arrieta G, Máttar S, Barguil A, Tamayo L, Padilla T, Bedoya Z, et al. 2004. Seroprevalencia de leptospirosis porcina en el departamento de Córdoba. Rev Colomb Cienc Pec 17: 141-147.

3. Baer AA, Miller MJ, Dilger AC. 2013. Pathogens of interest to the pork industry: a review of research on interventions to assure food safety. Compr Rev Food Sci F 12: 183-217. doi: 10.1111/15414337.12001

4. Bonardi S, Bruini I, D'Incau B, Van Damme I, Carniel E, Brémont S, Cavallini P, et al. 2016. Detection, seroprevalence and antimicrobial resistance of Yersinia enterocolitica and Yersinia pseudotuberculosis in pig tonsils in Northern Italy. Int $\mathrm{J}$ Food Microbiol 235: 125-132. doi: 10.1016/ j.ijfoodmicro.2016.07.033

5. Bonde M, Sorensen JT. 2012. Faecal Salmonella shedding in fattening pigs in relation to the presence of Salmonella antibodies in three pig production systems. Livest Sci 150: 236-239. doi: 10.1016/j.livsci.2012.09.002

6. Calderón A, Rodríguez V, Máttar S, Arrieta G. 2014. Leptospirosis in pigs, dogs, rodents, humans, and water in an area of the Colombian tropics. Trop Anim Health Pro 46: 427-432. doi: 10.1007/ s11250-013-0508-y

7. Casas G. 2013. Protocolo toma de muestra de sangre en porcinos. Universidad Nacional de Colombia. Facultad de Medicina Veterinaria y Zootecnia. [Internet]. Disponible en: http://medicina veterinaria y de zootec-nia.bogota. unal. edu.co/fileadmin/FVMZ/Servicios/ bioetica/Pro_autorizados/002_Protocolo_toma_muestra-_sangre_en_cerdos. pdf

8. [DANE] Dirección Nacional de Estadística. 2017. Encuesta de sacrificio de ganado. [Internet]. Disponible en: www.dane.gov.co/index.php/ estadisticas-por-tema/agropecuario/encuesta-de-sacrificio-de-ganado 
9. Dechner A. 2014. A retrospective analysis of the leptospirosis research in Colombia. J Infect Dev Countr 8: 258264. doi: $10.3855 /$ jidc. 3123

10. Díaz, M, Mendoza E, Linares C, Gasca H, Jaramillo D, Barón J, Botero A, et al. 2014. Colombia, Sanidad animal 2014. [Internet]. Disponible en: www.ica.gov.co/getattachment/ 986 d d 783 - 8 f 37 - 4 a b 3 - b c 33 39995bd8c065/2014.aspx

11. [FAO] Organización de las Naciones Unidas para la Alimentación y la Agricultura. 2014a. Cerdos y riesgos para la salud pública. [Internet]. Disponible en: www.fao.org/ag/againfo/ themes/es/pigs/HH_risks.html

12. [FAO] Organización de las Naciones Unidas para la Alimentación y la Agricultura. 2014b. Producción y sanidad. [Internet]. Disponible en: www.fao.org/ag/againfo/themes/es/pigs/ home.html

13. [FAO] Food and Agriculture Organization of the United Nations, [WHO] World Health Organization. 2014. Multicriteria-based ranking for risk management of food-borne parasites. Microbiological Risk Assessment Series 23. [Internet]. Disponible en: www.who.int/foodsafety/publications/ mra_23/en/

14. Feraud D, Abeledo G 2005. Primer reporte en Cuba de Leptospira interrogans serovar Tarassovi y caracterización clínica epizootiologica en focos de leptospirosis porcina. REDVET 6(4). [Internet]. Disponible en: http:// www.veterinaria.org/revistas/redvet/ n040405/040502.pdf

15. Framstad T, Sjaastad Ø, Aass RA. 2000. Bleeding and intravenous techniques in pigs. Norwegian School of Veterinary Science and Norwegian Independent Meat Association. Oslo. [Internet]. Disponible en: http:// oslovet.norecopa.no/teaching/pig/ pigbleed
16. García-González R, Reyes-Torres A, Basilio-Hernández D, Ramírez-Pérez M, Rivas-Sánchez B. 2013. Leptospirosis un problema de salud pública. Rev Latinoam Patol Clin 60: 57-70.

17. Gradassi M, Caminiti A, Galletti G, Santi A, Paternoster G, Tamba M, Zanoni M, et al. 2015. Suitability of a Salmonella control programme based on serology in slaughter heavy pigs. Res Vet Sci 101: 154-160. doi: 10.1016/j.rvsc.2015.06.015

18. Henao JS, Ramírez, E, Rondón-Barragán IS. 2012. Análisis de las Buenas Prácticas de Producción en granjas porcícolas del departamento del Tolima $\mathrm{y}$ factores de riesgo asociados a la presencia de Salmonella spp. Rev CES Med Zootec 7: 11-20.

19. Hernández M, Gómez-Laguna J, Tarradas C, Luque I, García-Valverde R, Reguillo L, Astorga RJ. 2014. A serological survey of Brucella spp, Salmonella spp, Toxoplasma gondii and Trichinella spp in Iberian fattening pigs reared in free-range systems. Transbound Emerg Dis 61: 477-481. doi: 10.1111/tbed.12049

20. Herrero L, Gracia MJ, PérezArquillué $C$, Lázaro $R$, Herrera $M$, Herrera A, Bayarri S. 2016. Toxoplasma gondii: pig seroprevalence, associated risk factors and viability in fresh pork meat. Vet Parasitol 224: 5259. doi: 10.1016/j.vetpar.2016.05.010

21. Hill DE, Chirukandoth S, Dubey JP, Lunney JK, Gamble HR. 2006. Comparison of detection methods for Toxoplasma gondii in naturally and experimentally infected swine. Vet Parasitol 141: 9-17. doi: 10.1016/ j.vetpar.2006.05.008

22. INS-Sivigila. 2016. Boletín epidemiológico nacional. Semana epidemiológica número 52: 25 Diciembre - 31 Diciembre.[Internet]. Disponible en: https:// www.ins.gov.co/buscador eventos/Boletin Epidemiologico/2016\%20Bolet\% C $3 \%$ A D n $\% 2$ - 0 e p i d e m i o $1 \%$ C3\%B3gico\%20semana\%2052\%20-.pdf 
23. Laverde LM, Builes LM, Masso CJ. 2009. Detección de Trichinella spiralis en cerdos faenados en dos plantas de beneficio en el municipio de Bello. Revista CES 4: 47-56.

24. Lehmann T, Graham DH, Dahl E, Sreekumar C, Launer F, Corn JL, Gamble HR, Dubey JP. 2003. Transmission dynamics of Toxoplasma gondii on a pig farm. Infect Genet Evol 3: 135-141.

25. Ochoa JE, Sánchez A, Ruiz I. 2000. Epidemiología de la leptospirosis en una zona andina de producción pecuaria. Rev Panam Salud Publ 7: 325-331

26. [OMS] Organización Mundial de la Salud. 2008. Human leptospirosis: guidance for diagnosis, surveillance and control. [Internet]. Disponible en: http:// apps.who.int/iris/bitstream/10665/42667/ 1/WHO_CDS_CSR_EPH_2002.23.pdf

27. Ortega-Pacheco A, Acosta Viana KY, Guzmán-Marín E, Segura-Correa JC, Alvarez-Fleites M, Jiménez-Coello M. 2013. Prevalence and risk factors of Toxoplasma gondii in fattening pigs farm from Yucatán, Mexico. Biomed Res Int 2013 : 231497. doi: 10.1155/2013/231497

28. Picardeau M. 2013. Diagnosis and epidemiology of leptospirosis. Med Maladies Infect 43: 1-9. doi: 10.1016/ j.medmal.2012.11.005

29. Pozio E. 2007. World distribution of Trichinella spp infections in animals and humans. Vet Parasitol 149: 3-21. doi: 10.1016/j.vetpar.2007.07.002

30. Romero-Vivas CM, Thiry D, Rodríguez V, Calderón A, Arrieta G, Máttar S, Cuello M, et al. 2013. Molecular serovar characterization of Leptospira isolates from animals and water in Colombia. Biomedica 33(Suppl 1): 179-184.
31. Solaymani-Mohammadi S, Petri WA. 2006. Zoonotic implications of the swinetransmitted protozoal infections. Vet Parasitol 140: 189-203. doi: 10.1016/ j.vetpar.2006.05.012

32. Surpat S, Pascu M, Gartner L, Brezovan D, Savici J, Morariu S, Herman V. 2014. Seroprevalence of Salmonella spp infection in pigs fattening from western Romania. J Biotechnol 185: S95 doi: 10.1016/ j.jbiotec.2014.07.324

33. Vado-Solís I, Cárdenas-Marrufo MF, Jiménez-Delgadillo B, Alzina-López A, Laviada-Molina H, Suarez-Solís V, Zavala-Velázquez JE. 2002. Clinicalepidemiological study of leptospirosis in humans and reservoirs in Yucatán, Mexico. Rev Inst Med trop SP 44: 335-340. doi: 10.1590/S0036-46652002000-600008

34. van der Giessen J, Fonville M, Bouwknegt M, Langelaar M, Vollema A. 2007. Seroprevalence of Trichinella spiralis and Toxoplasma gondii in pigs from different housing systems in The Netherlands. Vet Parasitol 148: 371-374. doi: 10.1016/j.vetpar.2007.06.009

35. van der Wolf PJ, Elbers AR, van der Heijden HM, van Schie $F W$, Hunneman WA, Tielen MJ. 2001. Salmonella seroprevalence at the population and herd level in pigs in The Netherlands. Vet Microbiol 82: 171-184. doi: 10.1016/S0378-1135(00)00387-4

36. Vanantwerpen G, Van Damme I, De Zutter L, Houf K. 2014. Seroprevalence of enteropathogenic Yersinia spp in pig batches at slaughter. Prev Vet Med 116: 193-196. doi: 10.1016/j.prevetmed.2014.05.011

37. von Altrock, Roesler U, Waldmann KH. 2011. Herd factors associated with the serological Yersinia prevalence in fattening pig herds. Foodborne Pathog Dis 8: 1249-1255. doi: 10.1089/ fpd.2011.0883 